\title{
Root of Angelica gigas Nakai ameliorates ischemic stroke-induced brain injury in mice through the activation of the PI3K/AKT/mTOR and MAPK pathways
}

\section{Se-Eun Lee}

Pusan National University School of Korean Medicine

Chiyeon Lim

Dongguk University College of Medicine

Suin Cho ( $\nabla$ sicho@pusan.ac.kr)

Pusan National University School of Korean Medicine https://orcid.org/0000-0001-8507-1382

\section{Research}

Keywords: Angelica gigas, ischemic stroke, infarction, edema, neuroprotection

Posted Date: April 13th, 2020

DOI: https://doi.org/10.21203/rs.3.rs-21816/v1

License: (c) (1) This work is licensed under a Creative Commons Attribution 4.0 International License.

Read Full License 


\section{Abstract}

Background: Ischemic stroke results in disability and increased risk of morbidity, resulting in a massive burden on patients and caregivers. Recombinant tissue plasminogen activator (r-tPA) has a limited window of opportunity for efficacy and causes side effects including hemorrhage; therefore, safer and more effective therapeutic strategies are required. In this study, the neuroprotective effects of a methanolic extract of AGR (AGmex) in ischemia/reperfusion-induced brain injury in mice were investigated.

Methods: Ischemic brain injury was induced in the mice by transient occlusion of the middle cerebral artery (tMCAO) for $120 \mathrm{~min}$, and the effect of AGmex on the amount of infarction was measured. Cresyl violet and hematoxylin and eosin stains were used to identify changes in the neurons, nuclei, and cytoplasm. Western blotting, immunohistochemistry, and terminal deoxynucleotidyl transferase dUTP nick end labeling staining were used to elucidate the neuroprotective mechanism of AGmex.

Results: AGmex effectively reduced the infarction volume when the mice were pre-treated at $1,000 \mathrm{mg} / \mathrm{kg}$ bw/day for two consecutive days (AGmex 1000 group). Neurons, nuclei, and cytoplasmic staining were the lowest in the MCAO group, but recovered in the AGmex 1000 group. In addition, proteins related to cell growth, differentiation, and death were up-regulated in the AGmex 1000 group.

Conclusion: The major recovery mechanisms appeared to be attenuation of the mitochondrial function of $\mathrm{Bcl}-2 / \mathrm{Bax}$, and activation of the PI3K/AKT/mTOR and MAPK signaling pathways in ischemic neurons.

\section{Background}

Cerebrovascular diseases (CVDs), caused by heart or blood vessel abnormalities, are a major cause of death worldwide. Of all of the CVDs, acute ischemic cerebral stroke is considered as the leading cause of morbidity and mortality in modern society $[1,2]$. When ischemic stroke occurs, cerebral inflammation and cell death are induced in the ischemic lesions, and inflammatory signals are activated by harmful stimuli such as arterial occlusion [3].

Thrombolytic agents have been used for the treatment of stroke with some success. However, there are limitations to their use, including side effects such as hemorrhage, and the limited window of opportunity for efficacious administration. Thus, further research on the prevention and treatment of ischemic stroke is in high demand [4].

The most commonly used mice model that temporarily block the middle cerebral artery (MCA) is known as the Koizumis' method [5]. In this study, we adapted the Koizumis' method with some modifications.

Angelica gigas Nakai, also called Korean angelica is a perennial plant from Asian countries, and its roots are used in traditional herbal medicine [6]. In Korea, the root of $A$. gigas is the source of Angelicae Gigantis Radix (AGR), while that of $A$. sinensis is used in China. 
According to traditional Korean Medicine, stroke is mainly caused by blood stasis, and AGR has been widely used by traditional medicine practitioners to treat ischemia related diseases [6].

Pharmacologically, it has been reported to have anticancer and antibacterial effects, antioxidant activity, and to improve circulatory diseases [7-9]. More recently, research has indicated that it has neuroprotective, anti-inflammatory, and antidementia effects (Additional File 1 as supplementary Table 1, and citations therein).

Therefore, in the present study, the neuroprotective potential of a methanol extract of AGR (AGmex) was assessed in male C57BL/ 6 mice, using a modified version of Koizumi's method, to investigate the medicinal application of AGR. Additionally, its proposed underlying molecular mechanisms of action are reported.

\section{Materials And Methods}

\section{Reagents}

Phosphate buffered saline (PBS) was purchased from Bio Basic Inc. (Markham, Ontario, Canada). 2,3,5Triphenyl-tetrazolium chloride (TTC), cresyl violet, Evans blue (EB), trichloroacetic acid, and propidium iodide (PI) were purchased from Sigma-Aldrich Co. (St. Louis, MO, USA). The optimal cutting temperature (OCT) compound cryostat embedding medium was purchased from Thermo Fisher Scientific (Waltham, MA, USA). Protein extraction solution was purchased from iNtRON (Seongnam-si, Gyeonggi-do, Korea). Primary antibodies for B-cell lymphoma 2 (Bcl-2), bcl-2-like protein 4 (Bax), phospho-phosphoinositide 3kinase (p-PI3K), protein kinase B (PKB, AKT), phospho-extracellular-signal-regulated kinase (p-ERK), ERK, phospho-c-Jun N-terminal kinase ( $p$-JNK), JNK, phospho-p38 mitogen-activated protein kinase (p-p38), p38, and manganese superoxide dismutase (MnSOD) were purchased from Cell Signaling Technology (Danvers, MA, USA). PI3K, p-AKT, phospho-mammalian target of rapamycin ( $\mathrm{p}-\mathrm{mTOR}$ ), sirtuin 1 (SIRT1), and $\beta$-actin were from Santa Cruz biotechnology Inc. (Dallas, TX, USA), and aquaporin 4 (AQP4) was from Abcam Inc. (Milton, Cambridge, UK). BCA reagent, bovine serum albumin (BSA) standard, and enhanced chemiluminescence $(E C L)$ western blotting chemiluminescent substrate were purchased from Thermo Fisher Scientific (Waltham, MA, USA).

\section{Preparation of the methanolic extract of AGR}

Dried AGR was purchased from a commercial supplier (Kwangmyung-Dang, Ulsan, Korea) and 12,800 g was extracted in methanol for $48 \mathrm{~h}$. The methanolic extract of AGR was evaporated using a rotary evaporator and lyophilized. The obtained lyophilized extract (AGmex) was stored at $-25^{\circ} \mathrm{C}$ in conical tubes. The AGR specimen (Specimen no. 2017-AGR-05) and AGmex (Voucher no. 2017Ex-AGR-05) were deposited $\left(4^{\circ} \mathrm{C}\right.$ and $-25^{\circ} \mathrm{C}$, respectively) at in the Plant and Extract Bank at Pusan National University School of Korean Medicine for future reference.

A commonly recommended clinical dose of AGR is $10 \mathrm{~g} / 60 \mathrm{~kg} \mathrm{bw} /$ day for adults. This is equivalent to 26 $\mathrm{mg} / \mathrm{kg}$ bw/day AGmex, which can be converted to $300 \mathrm{mg} / \mathrm{kg}$ bw/day for mice. Thus, based on the 
relevant clinical dosage of AGR, AGmex was orally administered to the mice daily at doses of 300 to $3,000 \mathrm{mg} / \mathrm{kg}$ bw/day.

\section{High performance liquid chromatographic (HPLC) analysis of AGR}

To obtain the fingerprint of the AGR used in this experiment, we determined the presence of nodakenin and decursin, the main components of AGR, using a high performance liquid chromatography (HPLC) system (Shimadzu, Kyoto, Japan) equipped with an LC-20AD pump, an SIL-20A autosampler, an SPDM20A detector, and a CTO-20A column oven. Samples were separated using a YMC-Triart $\mathrm{C}_{18}$ column. The mobile phase and elution system are shown in Fig. 1.

\section{Experimental schedule for dosing and transient middle cerebral artery occlusion (tMCAO)}

To evaluate the neuroprotective effect of AGmex, mice were subjected to tMCAO for 120 min after prior pre-treatment with AGmex. When the AGmex doses had been optimized, mice were post-treated with AGmex or nimodipine (ND) to compare their neuroprotective efficacies. ND was used as the reference agent as it has been used as a positive control in previous studies $[10,11]$.

Mice in the pre-treatment groups were orally administered one or two doses of 300-3,000 mg/kg bw AGmex either $24 \mathrm{~h}$ prior and/or $1 \mathrm{~h}$ prior to the tMCAO surgery. tMCAO was then performed for $120 \mathrm{~min}$. In the post-tMCAO treatment group, $1,000 \mathrm{mg} / \mathrm{kg}$ AGmex was administered $1 \mathrm{~h}$ after the end of the tMCAO. The dose of ND was $60 \mathrm{mg} / \mathrm{kg}$ bw based on the dose used in previous studies [10,11]. A schematic procedure of the experiment is shown in Fig. 2.

\section{Experimental animals and induction of tMCAO}

Ten week old C57BL/ 6 male mice (Samtako Bio, Osan, Korea), weighing 25-30 g, were used for all studies. All the procedures in this study were approved by the Ethics Committee for Animal Care and Use at Pusan National University (Approval No. PNU 2017-1759), certified by the Korean Association of Laboratory Animal Care. tMCAO was performed on the mice using a version of Koizumi's method modified in our laboratory. The details of the protocol and materials used are as reported previously [12].

\section{Measurement of neurological deficit score (NDS) and infarct area}

The NDS were evaluated using the protocol of Lee et al. [12]. TTC staining can reflect mitochondrial functioning and has been shown to be a reliable marker of ischemic volume after ischemia. Serial $1 \mathrm{~mm}$ coronal sections were stained with TTC solution and digitized, and the ischemic area and non-infarct tissue were outlined and assessed using an image analysis system (Digimizer, Ostend, Belgium).

\section{Cardiac perfusion and brain cryosection}

The mice were anesthetized by $\mathrm{CO}_{2}$ respiration, and an incision was made in the abdomen and the cardiac tissue was perfused with PBS. PBS and a $4 \%$ paraformaldehyde (PFA) solution were used for 
perfusion and fixation, respectively. Post-fixation, the brain was soaked in 10\% PFA containing 10-30\% sucrose for 3 days at $4{ }^{\circ} \mathrm{C}$, and then cryosectioned to a thickness of $30 \mu \mathrm{m}$ (Leica, Wetzlar, Germany) for staining.

\section{Nissl staining, and hematoxylin and eosin (H\&E) staining}

Frozen slides were thawed and allowed to dry sufficiently on a slide warmer, before being dipped into an ethanol:chloroform (1:1 vol $/ \mathrm{vol})$ solution overnight. The slides were placed in $0.1 \%$ cresyl violet solution for $10 \mathrm{~min}$, then incubated at $40^{\circ} \mathrm{C}$. Then, they were washed once with distilled water, before being sequentially placed in $95 \%$ and $100 \%$ ethyl alcohol for 5 min each, sealed with a cover slip and mounting solution, and observed under a microscope (Axio, Zeiss, Oberkochen, Germany). The density of the neuronal cells in the cortex area was measured using an ImageJ program (NIH, MD, USA). The H\&E staining procedures have been described previously [12].

\section{Blood-brain barrier (BBB) permeability}

The permeability of the BBB was determined by measuring the amount of EB solution according to a previously published protocol [13].

\section{Immunohistochemistry (IHC)}

Frozen slides were allowed to thaw and dry sufficiently on a slide warmer, before the brain sections were incubated with blocking serum ( $2 \% \mathrm{BSA})$ for $1 \mathrm{~h}$ at $25^{\circ} \mathrm{C}$, followed by incubation for $4^{\circ} \mathrm{C}$ overnight with the primary antibodies for p-mTOR and AQP4. After overnight incubation, secondary antibody was added and the slides were incubated for $1 \mathrm{~h}$ at $25^{\circ} \mathrm{C}$. After the sections were washed 3 times, they were stained for 5 min using the reagent in the Envision kit (DAKO K5007, CA, USA). The slides were sequentially placed in $95 \%$, then $100 \%$, ethyl alcohol for 1 min each, dipped in xylene and mounted, then observed under a microscope (Axio, Zeiss, Oberkochen, Germany).

\section{Western blot analysis}

The ipsilateral (left) hemispheric proteins from mice brain were isolated using a protein extraction solution. Lysates were obtained by centrifugation at $15,871 \times \mathrm{g}$ for $10 \mathrm{~min}$ at $4^{\circ} \mathrm{C}$. Protein supernatants were quantitated using a BSA standard method. Protein samples $(30 \mu \mathrm{g})$ were separated by SDS-PAGE (6$12 \%)$, then transferred onto PVDF membranes (Millipore, Darmstadt, Germany). The membranes were blocked using 5\% skimmed milk in TBST (Tris-buffered saline, $0.1 \%$ Tween 20 ) buffer for $1 \mathrm{~h}$ at $25^{\circ} \mathrm{C}$, and incubated at $4^{\circ} \mathrm{C}$ overnight with the primary antibodies. After overnight incubation, secondary antibodies were added for $1 \mathrm{~h}$ at $25^{\circ} \mathrm{C}$. After incubation, the membranes were treated with ECL solution and the expressed proteins were detected using a photosensitive luminescent analyzer system (Amersham ${ }^{\text {TM }}$ Imager 600, Buckinghamshire, UK). Band intensities were analyzed using an ImageJ program ( $\mathrm{NIH}, \mathrm{MD}$, USA), and are represented as the ratios of the densities of the respective phospho-form vs the total-form protein bands. 


\section{Terminal deoxynucleotidyl transferase dUTP nick endlabeling (TUNEL) staining}

Frozen slides were thawed and allowed to dry sufficiently on a slide warmer, before the sections were hydrated in PBS and permeabilized for $2 \mathrm{~min}$ in ethanol:acetic acid $(1: 1 \mathrm{vol} / \mathrm{vol})$ at $25^{\circ} \mathrm{C}$. A DeadEnd ${ }^{\text {tm }}$ Fluorometric TUNEL System (Promega Corp., WI, USA) protocol was used to visualize TUNEL-labeled nuclei. Tissue sections were counterstained with $1 \mu \mathrm{g} / \mathrm{ml} \mathrm{PI}$ to visualize the total number of nuclei. The stained sections were observed under a fluorescence microscope (Nikon Fluorescence Microscopy, Tokyo, Japan).

\section{Statistical analysis}

One-way analysis of variance (ANOVA) was used to determine the statistical differences between the mean values of the groups using the Sigmaplot version 12.0 program (Systat Software Inc., CA, USA). Data are expressed as mean \pm standard deviation (SD), and differences were considered statistically significant at $p<0.05$.

\section{Results}

\section{Analytical comparison of AGmex with its standards, nodakenin and decursin}

Nodakenin and decursin are the major pharmacologically active compounds found in A. gigas. Their structures and profile on an HPLC chromatogram are shown in Fig. 1A. The presence of nodakenin and decursin in AGmex was confirmed using HPLC (Fig. 1B).

\section{Measurements of infarct volume, edema area, and NDS}

To study the effect of AGmex, the mice were pre-treated with AGmex before induction of ischemic stroke. Doses of $300,1,000$, and $3,000 \mathrm{mg} / \mathrm{kg}$ bw were administered once ( $1 \mathrm{~h}$ prior to tMCAO) or twice ( $1 \mathrm{~h}$ prior to tMCAO and $24 \mathrm{~h}$ earlier). The most effective regimen was the double $1,000 \mathrm{mg} / \mathrm{kg} \mathrm{bw} /$ day dose (Fig. $3 \mathrm{~A})$. The total infarct volume percentile in the ipsilateral hemispheric brain in the mice treated twice with AGmex at $1,000 \mathrm{mg} / \mathrm{kg}$ bw/day was $15.56 \pm 2.22 \%$, and that of the tMCAO group alone was $51.12 \pm$ $9.24 \%$.

Since $1,000 \mathrm{mg} / \mathrm{kg}$ bw was found to be the optimal concentration of AGmex for pre-treatment, a dose of $1,000 \mathrm{mg} / \mathrm{kg}$ bw was used for the post-treatment study. Although a lower average total infarct volume percentile was seen in the mice post-treated with $1,000 \mathrm{mg} / \mathrm{kg}$ bw AGmex, this difference was not statistically significant. Nimodipine (ND), a drug used in the clinic to treat ischemic neuropathy at 6 $\mathrm{mg} / \mathrm{kg}$ bw/day, was administered to the mice in this study at an equivalent dose of $60 \mathrm{mg} / \mathrm{kg} \mathrm{bw}$. However, no effect on total infarct volume percentile was seen after oral administration of this dose of ND post-treatment in this study (Fig. 3A).

A significant reduction in the amount of brain edema was observed in the groups pre-treated with AGmex once at $300 \mathrm{mg} / \mathrm{kg}$ bw $\left(11.35 \pm 0.33 \mathrm{~mm}^{3}\right)$ and twice at $1,000 \mathrm{mg} / \mathrm{kg}$ bw/day $\left(2.27 \pm 2.97 \mathrm{~mm}^{3}\right)$, when 
compared with the amount of edema seen in the tMCAO alone group $\left(29.86 \pm 8.85 \mathrm{~mm}^{3}\right)$ (Fig. 3B).

No statistical changes were observed in the NDS between the MCAO-induced groups. However, in the ND group, used as a positive control in previous reports $[10,11]$, the NDS was significantly higher (at $3.0 \pm$ 0.0 ) than that seen in the MCAO group ( $2.0 \pm 0.0$ ) (Fig. $3 \mathrm{C}$ ), suggesting that ND may potentially have an adverse effect on post-stroke behavior.

During MCAO induction in the anesthetized mice, a laser Doppler probe was attached to the temporal surface of the skull. CCA occlusion resulted in the cerebral blood flow being reduced to approximately 68 $\pm 8 \%$ of baseline levels. When the MCA was occluded, the Doppler cerebral blood flow signal decreased to approximately $11 \pm 2 \%$ of the signal seen during total blood flow (Additional File 2 as supplementary Fig. 1). As previous studies have indicated that AGR promotes blood circulation, the rCBF in the AGmextreated group was expected to increase during the MCA occlusion period. However, no difference in rCBF was seen in any of the groups (Supplementary Fig. 1).

\section{Assessing changes of BBB permeability}

As $1,000 \mathrm{mg} / \mathrm{kg}$ bw/day over two days was found to be the most effective dose of AGmex in the pretreatment study, this dose was also used for the mechanistic studies.

EB leakage in the ipsilateral hemisphere in the TMCAO group was significantly increased when compared with that in the sham-operated group. In addition, in the group pre-treated with 1,000 $\mathrm{mg} / \mathrm{kg} \mathrm{bw} / \mathrm{day}$ AGmex (AGmex1000 group), the EB leakage was significantly lower than that seen in the tMCAO group. There was no significant difference in leakage in the contralateral hemisphere between the shamoperated group and the TMCAO group; however, it was significantly higher in the AGmex 1000 group than in the tMCAO group (Fig. 4A).

IHC staining in the ischemic lesions of the ipsilateral hemisphere showed that AQP4 was expressed mainly in the cortical neurons (red arrows indicate AQP4 positive stain, Fig. 4B). AQP4 expression was lower in the AGmex 1000 group than in the TMCAO group (black arrows indicate AQP4 negative stain, Fig. $4 B)$.

\section{Morphological changes to neuronal cells}

In the sham-operated group, cresyl violet staining indicated that the neuronal cells were intact, with a morphologically well-arranged cytoplasm and nucleus (black arrows, Fig. 5A). In the tMCAO group, the neuronal cells were apoptotic, with aberrant morphology (red arrows, Fig. 5A). In the AGmex-treated group, the cells were similar to those of the sham-operated group (black arrows, Fig. 5A). Cresyl violet staining, also known as Nissl staining, indicated that the cell density of the TMCAO-induced group was $77.3 \pm 9.08 \%$ of that of the sham-operated group, and that of the AGmex 1000 group was $98.28 \pm 3.63 \%$ (Fig. 5A). 
Cells of the tMCAO-induced group showed DNA damage, presenting as cell shrinkage, pyknotic nuclei, and eosinophilic cytoplasm (red arrows, Fig. 5B). However, those of the AGmex 1000 group appeared to be much less damaged, similar to those of the sham-operated group (black arrows, Fig. 5B). Compared with the sham-operated group, H\&E staining indicated that the density of the cells in the TMCAO-induced group was $79.61 \pm 9.23 \%$ and that of those in the AGmex 1000 group was $108.01 \pm 6.11 \%$ (Fig. 5B).

\section{Apoptotic changes to the ipsilateral cerebral cortex}

In the sham-operated group, neuronal cells were PI-stained and evenly distributed, and no TUNEL-positive cells were observed. However, in the TMCAO group, TUNEL-positive cells were clearly visible and highly stained, with more condensed PI staining, indicating tMCAO-induced apoptotic changes. On the contrary, cells of the AGmex 1000 group showed nuclear condensation in the PI staining, but TUNEL-positive cells were not detected, suggesting that AGmex has an anti-apoptotic effect (Fig. 6A).

\section{Expression of cell death-related molecules in the ipsilateral cerebral cortex}

Protein expression levels are shown in Fig. 7A. Bcl-2 and Bax proteins are related to typical apoptosis signaling that occurs in mitochondria $[14,15]$. The Bcl-2/Bax expression ratio was $1.00 \pm 0.04$ in the sham-operated group, $0.75 \pm 0.02$ in the tMCAO group, and $0.91 \pm 0.09$ in the AGmex 1000 group (Fig. 7B). These results indicate that apoptosis was observed in the $\mathrm{MCAO}$ group, but that administration of $1,000 \mathrm{mg} / \mathrm{kg}$ bw/day AGmex on two consecutive days prior to the tMCAO protected the neuronal cells from apoptosis.

The PI3K/AKT/mTOR signaling pathway regulates a variety of cellular functions, including differentiation, proliferation, survival, and autophagy $[15,16]$. Expression of mTOR protein was evaluated using IHC staining (Fig. 6B). It was over-expressed in the tMCAO group (red arrows), and the expression was down-regulated in the AGmex 1000 group.

The ratio of $\mathrm{p}-\mathrm{PI} 3 \mathrm{~K} / \mathrm{PI} 3 \mathrm{~K}$ expression was $1.00 \pm 0.05$ in the sham-operated group, $0.02 \pm 0.02$ in the tMCAO group, and $0.19 \pm 0.12$ in AGmex 1000 group (Fig. 7C). The ratio of $p$-AKT/AKT expression was $1.00 \pm 0.07$ in the sham-operated group, $0.70 \pm 0.16$ in the tMCAO group, and $1.18 \pm 0.06$ in the AGmex 1000 group (Fig. 7D).

MAPK signaling was activated in the tMCAO group (Fig. 7E, F, and G). ERK, one of the major signaling cassettes of MAPK, is related to cell growth and differentiation [17]. ERK signaling was activated in the AGmex 1000 group. The ratio of $p$-ERK/ERK expression was $1.00 \pm 0.11$ in the sham-operated group, 1.24 \pm 0.09 in the $\mathrm{TMCAO}$ group, and $1.91 \pm 0.40$ in the AGmex 1000 group (Fig. 7E). JNK activates apoptotic pathways by up-regulating pro-apoptotic genes $[17,18]$, and its increased expression in the tMCAO group was inhibited by AGmex administration. The ratio of $p-J N K / J N K$ expression was $1.00 \pm 0.11$ in the shamoperated group, $1.32 \pm 0.15$ in the tMCAO group, and $0.66 \pm 0.08$ in the AGmex 1000 group (Fig. 7F). Signaling pathway $\mathrm{p} 38$ regulates apoptosis, targeting members of the Bcl-2 family of proteins $[17,18]$. Its expression was up-regulated in the IMCAO group, but was even higher in the AGmex 1000 group. The 
ratio of p-p38/p38 expression was $1.00 \pm 0.28$ in the sham-operated group, $1.58 \pm 0.16$ in the tMCAO group, and $2.28 \pm 0.29$ in the AGmex 1000 group (Fig. 7G).

Several researchers have reported that herbal medicine or plant-derived compounds attenuate cerebral ischemic injury in a SIRT1-dependent manner $[19,20]$. However, in this study, neither tMCAO or AGmex administration had any significant effect on SIRT1 expression (Fig. 7H).

MnSOD, also known as superoxide dismutase 2, is specifically localized to the inner mitochondrial membrane, and has been reported to be related to antioxidant pathways [21-23], demonstrating neuroprotective effects against oxidative stress [24-26]. This suggests that it may be a potential target of herbal agents that have an effect on ischemic brain injury. In this study, tMCAO significantly increased the expression of MnSOD protein (relative expression levels were $1.00 \pm 0.16$ in the sham-operated group compared with $1.46 \pm 0.10$ in the $\mathrm{MCAO}$ group), and AGmex administration significantly inhibited this increase (1.04 \pm 0.19 in the AGmex 1000 group) (Fig. 7I).

\section{Discussion}

AGR and its related compounds have been reported to have neuroprotective, anti-inflammatory, reactive gliosis-suppressing, antidementia, antifungal, antiplatelet, anticoagulation, antioxidant, and anticancer effects in in vivo and in vitro (Additional File 1).

AGmex, the methanolic extract of AGR, reduced the total infarct volume and edema induced by ischemic stroke. The most efficient dosage was $1,000 \mathrm{mg} / \mathrm{kg}$ bw/day of AGmex, administered for two consecutive days before the TMCAO operation (Fig. 3). We used ND as the reference agent, based on previous reports on its potential in ischemic stroke [11]. However, in this study it did not have a positive effect. It is interesting to note that the average NDS of the mice given ND after the TMCAO operation was higher than that of those in the TMCAO group, suggesting that care should be taken when determining the dose of ND administered to patients with ischemic brain diseases.

AQP4 is known to be important in maintaining the brain water balance by regulating the integrity of the BBB. Brain edema is a major target for the treatment of human ischemic stroke $[27,28]$. In this study, AGmex attenuated ischemia-induced cerebral injury by regulation of AQP4 protein expression (Fig. 4).

The effect of AGmex pre-treatment on MCAO-induced cerebral damage was also assessed histologically. The cresyl violet stain for neurons and the H\&E stain for nuclei and cytoplasm showed that AGmex pretreatment resulted in improved cell density and morphology when compared with that seen after tMCAO alone (Fig. 5).

As shown in Fig. 6A, apoptotic cells were present in the tMCAO group, but their numbers were reduced by AGmex pre-treatment. Since mTOR kinase is an important regulator of cell growth, proliferation, and survival $[15,16]$, mTOR protein was over-expressed in both the tMCAO group and the AGmex 1000 group, suggesting the possibility of an MCAO stress-induced increase in cell proliferation signaling (Fig. 6B). 
These results indicate that $A G m e x$ inhibited the MCAO-induced apoptosis signaling, but did not interfere with the MCAO-induced cell survival signal.

PI3K activates AKT, a protein also involved in the differentiation and survival of cells [16]. In this study, these signals were up-regulated in the MCAO-induced mouse brain after pre-treatment of the mice with AGmex (Fig. 7B, C, and D).

MAPK is an intracellular signaling molecule with effects on cell migration, proliferation, and differentiation, and its relevant sub-molecules are ERK, JNK, and p38 [29]. In this study, expression levels of ERK, JNK, and p38 were increased by MCAO. AGmex pre-treatment reduced the expression of JNK, but increased the expression of ERK and p38 (Fig. 7E, F, and G). Overall, tMCAO promoted the MAPK pathway.

There have been many reports that SIRT1 can be a target of herbal medicines [30]. However, in this study neither tMCAO or the pre-administration of AGmex had any effect on SIRT1 expression (Fig. 7H).

As MnSOD appears to be neuroprotective against oxidative stress [21, 23], it is an important potential target for herbal agents which have positive effects on ischemic brain injury. In this study, tMCAO significantly increased the expression of MnSOD protein and AGmex significantly inhibited its increase. This suggests that antioxidative signals were activated by the damage caused by the $\mathrm{MCAO}$, and that the decrease in cerebral damage affected by pre-treatment with AGmex resulted in the inhibition of MnSOD protein expression (Fig. 7l).

In summary, mice dosed with AGmex at $1,000 \mathrm{mg} / \mathrm{kg}$ bw/day for two consecutive days before the tMCAO operation had less neuronal cell death than those that were not dosed with AGmex prior to the operation. This protective effect involved anti-edema and anti-apoptotic pathways, demonstrating attenuation of BBB disruption and activation of mitochondria-related cell survival signals. Overall, AGmex appeared to protect neurons and inhibit the apoptosis seen in MCAO-induced cerebral cell death and the mechanisms and pathways involved in the neuroprotective effects of AGmex could be summarized as Fig. 8.

\section{Conclusions}

AGR improved mitochondrial function via the Bcl-2/Bax, PI3K/AKT/mTOR, and MAPK signaling pathways in ischemic neurons, indicating that AGR can activate proteins involved in cell survival and inhibit those involved in cell death. This study provides preclinical evidence for the neuroprotective use of AGR in the treatment of ischemic stroke.

\section{Abbreviations}

AGR: Angelicae Gigantis Radix; BBB: blood-brain barrier; CVDs: cerebrovascular diseases; EB: Evans blue; ECL: enhanced chemiluminescence; MCA: middle cerebral artery; mTOR: mammalian target of rapamycin; ND: nimodipine; NDS: neurological deficit score; OCT: optimal cutting temperature; rCBF: relative cerebral 
blood flow; SIRT1: sirtuin 1; tMCAO: transient middle cerebral artery occlusion; TTC: 2,3,5-Triphenyltetrazolium chloride; TUNEL: terminal deoxynucleotidyl transferase dUTP nick endlabeling

\section{Declarations}

\section{Acknowledgment}

We gratefully acknowledge the technical assistance of Miss S.Y. Jeong of Kwangmyung-Dang Pharmaceuticals (Ulsan, Korea) for the HPLC analysis protocols.

\section{Authors' contributions}

SEL and SC designed the study. SEL and SC performed the experiments. CL conducted statistical analysis. SC wrote the manuscript. All authors read and approved the final version of the manuscript.

\section{Funding}

Not applicable.

\section{Availability of data and materials}

Please contact corresponding authors for data requests.

\section{Ethics approval and consent to participate}

All procedures in this study were approved and supervised by Ethics Committee for Animal Care and Use at Pusan National University (Approval No. PNU 2017-1759), certified by the Korean Association of Laboratory Animal Care.

\section{Consent for publication}

Not applicable.

\section{Declaration of Competing Interest}


The authors declare that they have no conflicts of interest.

\section{References}

1. Mittal SH, Goel D. Mortality in ischemic stroke score: A predictive score of mortality for acute ischemic stroke. Brain Circ. 2017;3(1):29-34.

2. Shah B, Bartaula B, Adhikari J, Neupane HS, Shah BP, Poudel G. Predictors of in-hospital mortality of acute ischemic stroke in adult population. J Neurosci Rural Pract. 2017;8(4):591-4.

3. Sims NR, Muyderman H. Mitochondria, oxidative metabolism and cell death in stroke. Biochim Biophys Acta. 2010;1802(1):80-91.

4. Hilbrich L, Truelsen T, Yusuf S. Stroke and cardiovascular diseases: the need for a global approach for prevention and drug development. Int J Stroke. 2007;2(2):104-8.

5. Koizumi JY, Nakazawa T, Ooneda G. Experimental studies of ischemic brain edema. I. A new experimental model of cerebral embolism in rats in which recirculation can be introduced in the ischemic area. Jap J Stroke. 1986;8:1-8.

6. Shin YJ, Park YK. Effects of different parts of Angelica gigas Nakai on brain damages and neuronal death in transient middle artery occlusion/reperfusion-induced ischemic rats. Kor J Herbol. 2014;29:85-93.

7. Joo SS, Park D, Shin S, Jeon JH, Kim TK, Choi YJ, Lee SH, Kim JS, Park SK, Hwang BY, Lee DI, Kim Y. Anti-allergic effects and mechanisms of action of the ethanolic extract of Angelica gigas in dinitrofluorobenzene-induced inflammation models. Environ Toxicol Pharmacol. 2010;30(2):127-33.

8. Park SJ, Jung JM, Lee HE, Lee YW, Kim DH, Kim JM, Hong JG, Lee CH, Jung IH, Cho Y, Jang DS, Ryu $\mathrm{JH}$. The memory ameliorating effects of INM-176, an ethanolic extract of Angelica gigas, against scopolamine- or A 1 1-42-induced cognitive dysfunction in mice. J Ethnopharmacol. 2012;143(2):611-20.

9. Seong G, Baek M, Lee Y, Won J. Quality evaluation of Angelica gigas Nakai with different drying methods and different root parts. Kor J Herbol. 2018;33:85-91.

10. Li JH, Lu J, Zhang H. Functional recovery after scutellarin treatment in transient cerebral ischemic rats: A pilot study with (18) F-fluorodeoxyglucose microPET. Evi Based Complement Alternat Med. 2013;2013:507091.

11. Zhao Q, Wang X, Chen A, Cheng X, Zhang G, Sun J, Zhao Y, Huang Y, Zhu Y. Rhein protects against cerebral ischemic/reperfusion-induced oxidative stress and apoptosis in rats. Int $\mathrm{J}$ Mol Med. 2018;41(5):2802-12.

12. Lee SE, Lim C, Lee M, Kim CH, Kim H, Lee B, Cho S. Assessing neuroprotective effects of Glycyrrhizae Radix et Rhizoma extract using a transient middle cerebral artery occlusion mouse model. J Vis Exp. 2018;142.

13. Manaenko A, Chen H, Kammer J, Zhang JH, Tang J. Comparison Evans blue injection routes: Intravenous versus intraperitoneal, for measurement of blood-brain barrier in a mice hemorrhage 
model. J Neurosci Methods. 2011;195(2):206-10.

14. Cao G, Pei W, Lan J, Stetler RA, Luo Y, Nagayama T, Graham SH, Yin XM, Simon RP, Chen J. Caspaseactivated DNase/DNA fragmentation factor 40 mediates apoptotic DNA fragmentation in transient cerebral ischemia and in neuronal cultures. Neurosci. 2001;21(13):4678-90.

15. Guo C, Yang M, Jing L, Wang J, Yu Y, Li Y, Duan J, Zhou X, Li Y, Sun Z. Amorphous silica nanoparticles trigger vascular endothelial cell injury through apoptosis and autophagy via reactive oxygen species-mediated MAPK/Bcl-2 and PI3K/Akt/mTOR signaling. Int J Nanomedicine. 2016;11:5257-76.

16. Prasad SS, Russell M, Nowakowska M. Neuroprotection induced in vitro by ischemic preconditioning and postconditioning: modulation of apoptosis and PI3K-Akt pathways. J Mol Neurosci. 2011;43(3):428-42.

17. Plotnikov A, Zehorai E, Procaccia S, Seger R. The MAPK cascades: signaling components, nuclear roles and mechanisms of nuclear translocation. Biochim Biophys Acta. 2011;1813(9):1619-33.

18. Chuang SM, Liou GY, Yang JL. Activation of JNK, p38 and ERK mitogen-activated protein kinases by chromium $(\mathrm{VI})$ is mediated through oxidative stress but does not affect cytotoxicity. Carcinogenesis. 2000;21(8):1491-500.

19. Zheng XW, Shan CS, Xu QQ, Wang Y, Shi YH, Wang Y, Zheng GQ. Buyang Huanwu decoction targets SIRT1/VEGF pathway to promote angiogenesis after cerebral ischemia/reperfusion injury. Front Neurosci. 2018;12:911.

20. Zhu HR, Wang ZY, Zhu XL, Wu XX, Li EG, Xu Y. Icariin protects against brain injury by enhancing SIRT1-dependent PGC-1alpha expression in experimental stroke. Neuropharmacology. 2010;59(12):70-6.

21. Ambe K, Watanabe $H$, Takahashi $S$, Nakagawa T. Immunohistochemical localization of Nox1, Nox4 and Mn-SOD in mouse femur during endochondral ossification. Tissue Cell. 2014;46(6):433-8.

22. Kinoshita M, Sakamoto T, Kashio A, Shimizu T, Yamasoba T. Age-related hearing loss in Mn-SOD heterozygous knockout mice. Oxi Med Cell Longev. 2013;2013:325702.

23. Lu X, Wang C, Liu B. The role of Cu/Zn-SOD and Mn-SOD in the immune response to oxidative stress and pathogen challenge in the clam Meretrix meretrix. Fish Shellfish Immunol. 2015;42(1):58-65.

24. Garnier P, Demougeot C, Bertrand N, Prigent-Tessier A, Marie C, Beley A. Stress response to hypoxia in gerbil brain: HO-1 and Mn SOD expression and glial activation. Brain Res. 2001;893(1-2):301-9.

25. Jung JE, Kim GS, Narasimhan P, Song YS, Chan PH. Regulation of Mn-superoxide dismutase activity and neuroprotection by STAT3 in mice after cerebral ischemia. J Neurosci. 2009;29(21):7003-14.

26. Scorziello A, Santillo M, Adornetto A, Dell'aversano C, Sirabella R, Damiano S, Canzoniero LM, Renzo GF, Annunziato L. NO-induced neuroprotection in ischemic preconditioning stimulates mitochondrial Mn-SOD activity and expression via Ras/ERK1/2 pathway. J Neurochem. 2007;103(4):1472-80.

27. Badaut J, Fukuda AM, Jullienne A, Petry KG. Aquaporin and brain diseases. Biochem Biophys Acta. 2014;1840(5):1554-65. 
28. Wang WW, Xie CL, Zhou LL, Wang GS. The function of aquaporin4 in ischemic brain edema. Clin Neurol Neurosurg. 2014;127:5-9.

29. Sun J, Nan G. The mitogen-activated protein kinase (MAPK) signaling pathway as a discovery target in stroke. J Mol Neurosci. 2016;59(1):90-8.

30. He W, Wang H, Zhao C, Tian X, Li L, Wang H. Role of liraglutide in brain repair promotion through Sirt1-mediated mitochondrial improvement in stroke. J Cell Physiol. 2020;235(3):2986-3001.

\section{Figures}
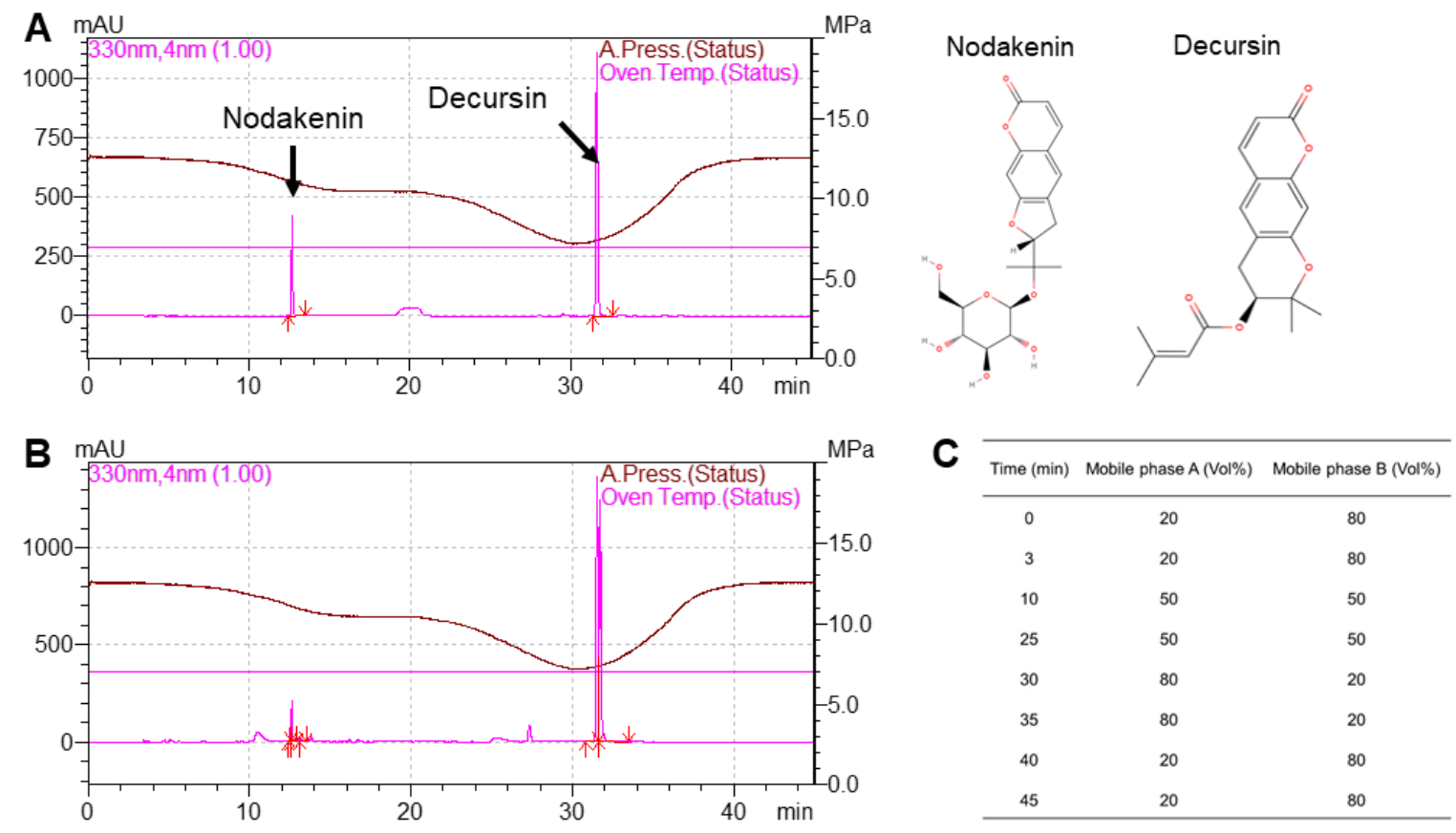

\section{Figure 1}

High performance chromatography (HPLC) of Angelicae Gigantis Radix (AGR) and its standards, nodakenin and decursin. A, HPLC chromatogram profile of the standards and their chemical structures. B, HPLC chromatogram profile of the AGR extract (AGmex). C, Mobile phase gradient (mobile phase A, acetonitrile; mobile phase B, water). Conditions used: HPLC, Shimadzu system (Shimadzu, Kyoto, Japan); column, YMC-Triart C18; wavelength, $330 \mathrm{~nm}$; column temperature, $35^{\circ} \mathrm{C}$; flow rate, $1 \mathrm{~mL} / \mathrm{min}$; injection volume, $10 \mu \mathrm{L}$. 


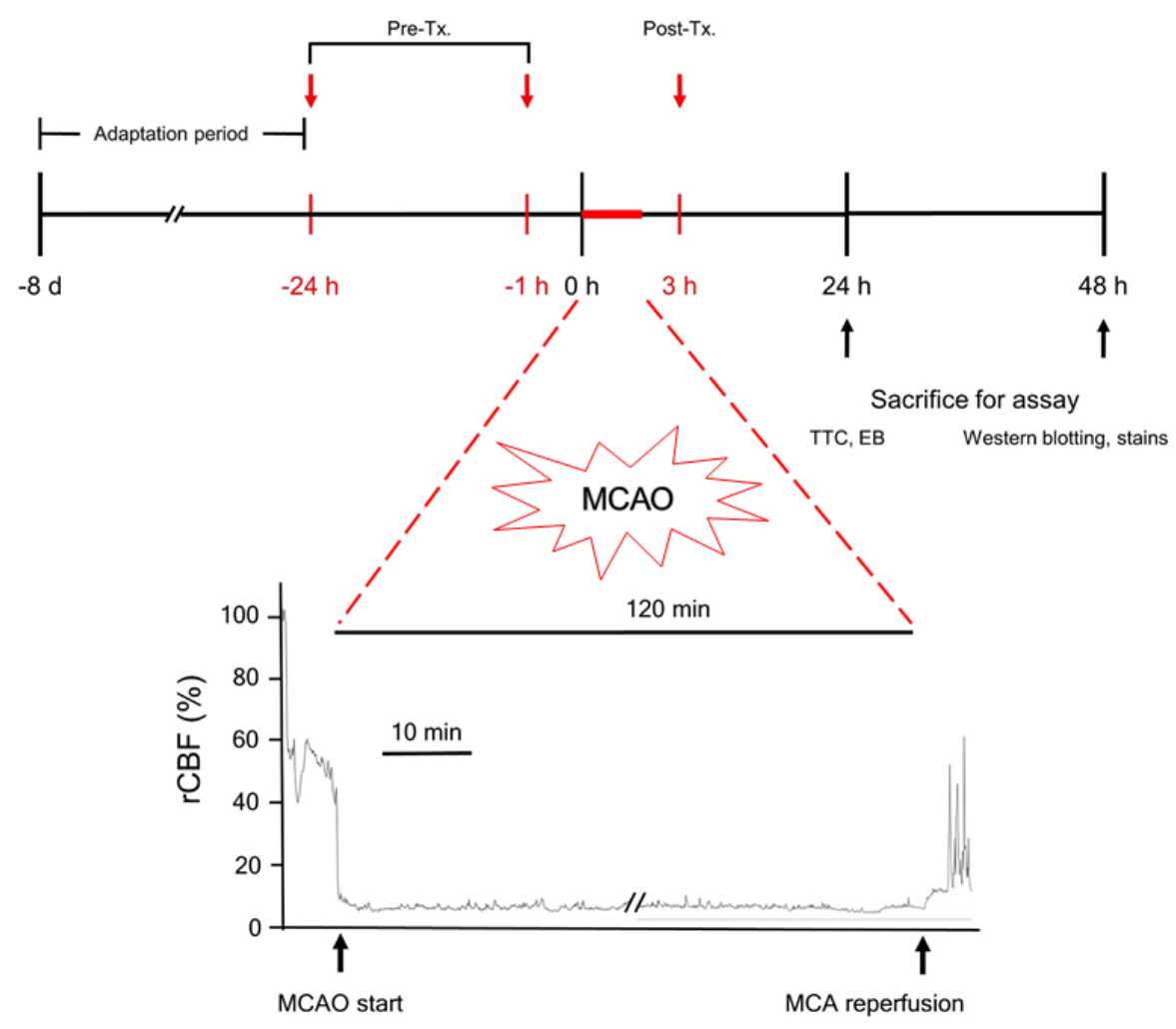

Figure 2

Experimental schedule of the 120 min transient middle cerebral artery occlusion (tMCAO) model. The mice had a one week adaptation period in the animal facility prior to the start of the study. Pre-treatment (Pre-Tx.) and post-treatment (Post-Tx.) studies were carried out to ascertain the effects of a methanolic extract of AGR (AGmex). TTC; 2,3,5-triphenyl-tetrazolium chloride; EB; Evans blue. 

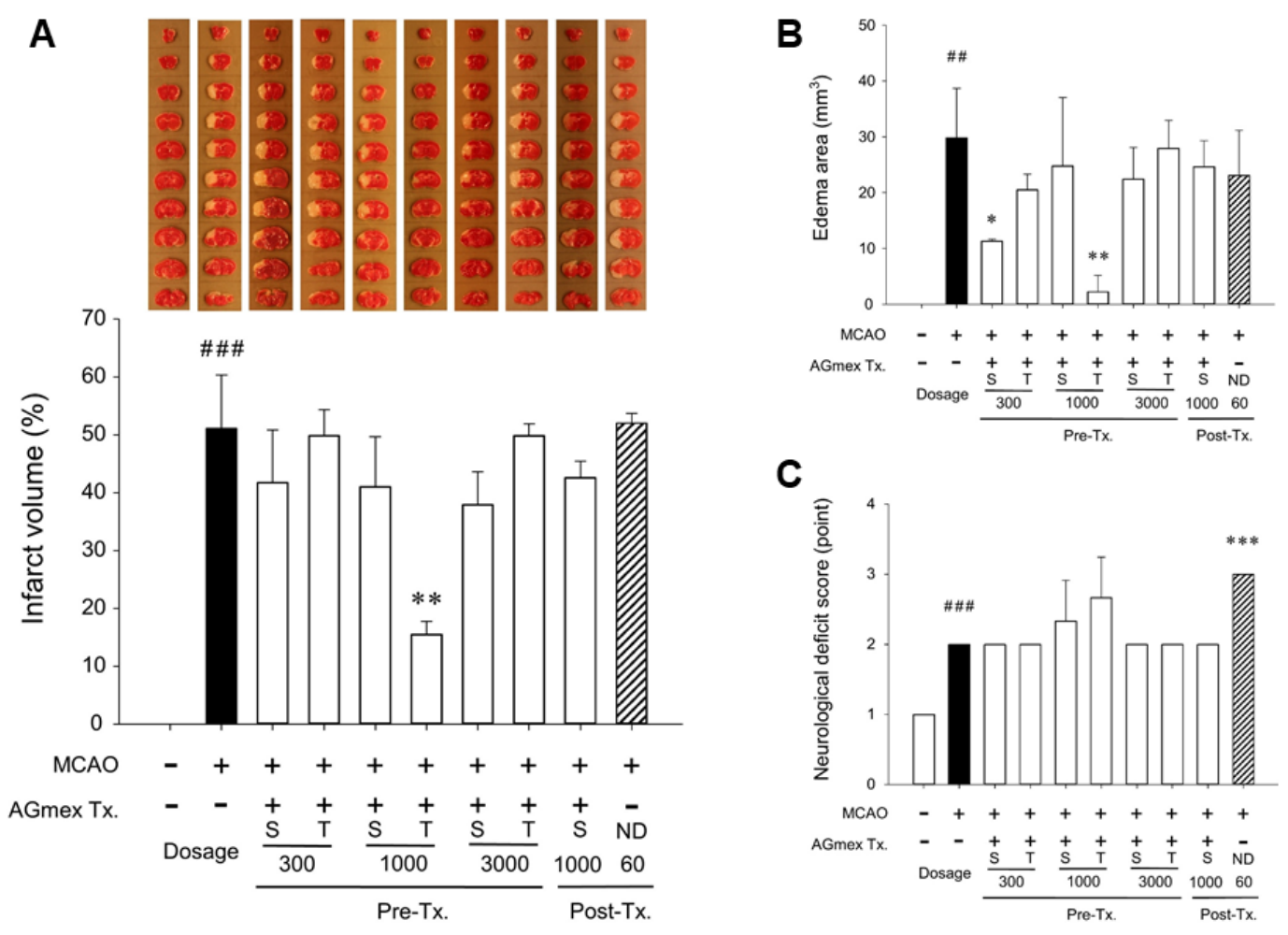

\section{Figure 3}

Measurements of infarct volume, edema area, and neuronal deficit scores (NDS). A, Representative photographs of TTC-stained brain slices $(1 \mathrm{~mm})$ showing the infarct area $24 \mathrm{hr}$ after tMCAO (upper column), and quantitative analysis of the total infarct volume (lower column). B, Quantitative analysis of the total edema area. C, Neurological behavioral evaluation (NDS). S, single AGmex treatment; T, AGmex treatment for two consecutive days; 300,1000 or $3000,300,1,000$ or $3,000 \mathrm{mg} / \mathrm{kg}$ bw/day AGmex; ND, nimodipine (reference agent); $60,60 \mathrm{mg} / \mathrm{kg}$ bw/day ND. All data are expressed as mean $\pm \mathrm{SD}(\mathrm{n}=3)$. \#\# $p<0.01$, \#\#\# $p<0.001$ vs. sham-operated group; $* p<0.05,{ }^{\star *} p<0.01, \star \star \star ~ p<0.001$ vs. MCAO group. 
A
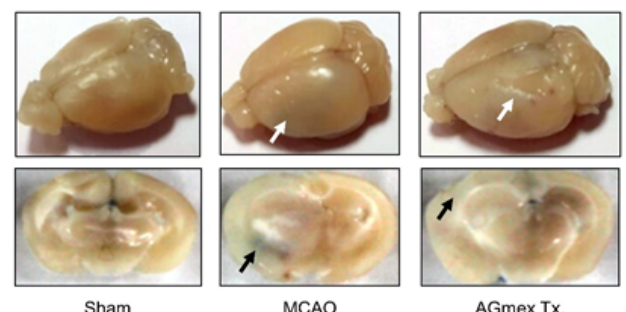

Ipsilateral hemisphere Contralateral hemisphere

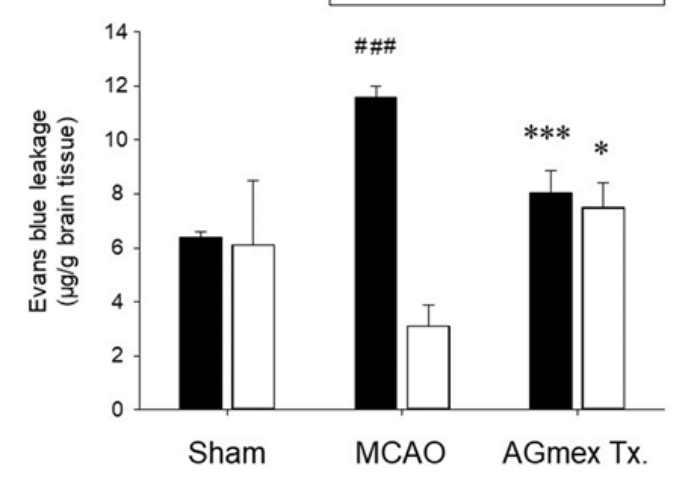

B

Sham

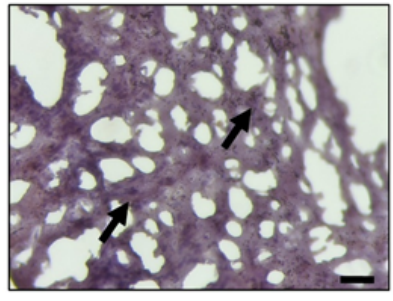

AGmex Tx.
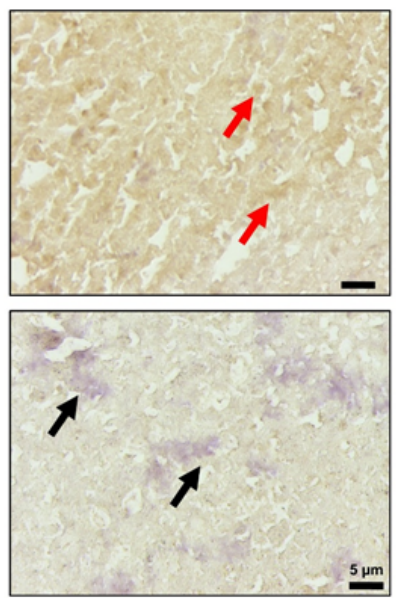

\section{Figure 4}

The effects of AGmex on the permeability of the blood-brain barrier (BBB) and on the localization of aquaporin 4 protein (AQP4) in the MCAO-induced mouse brain. A, Representative images of EB extravasation in a whole brain and coronal sections (upper column), and quantification of EB leakage in the ipsilateral (left) hemisphere and contralateral (right) hemisphere of the brain (lower column, $n=3$ ). B, Immunohistochemical (IHC) staining of AQP4 protein in the cerebral cortex (black arrows indicate AQP4 negative, red arrows, AQP4 positive, respectively. Scale bars: $5 \mu \mathrm{m}$. All data are expressed as mean \pm SD $(n=3)$. \#\#\# $p<0.001$ vs. sham-operated group; * $p<0.05$, and $* \star \star ~ p<0.001$ vs. MCAO group. 
A
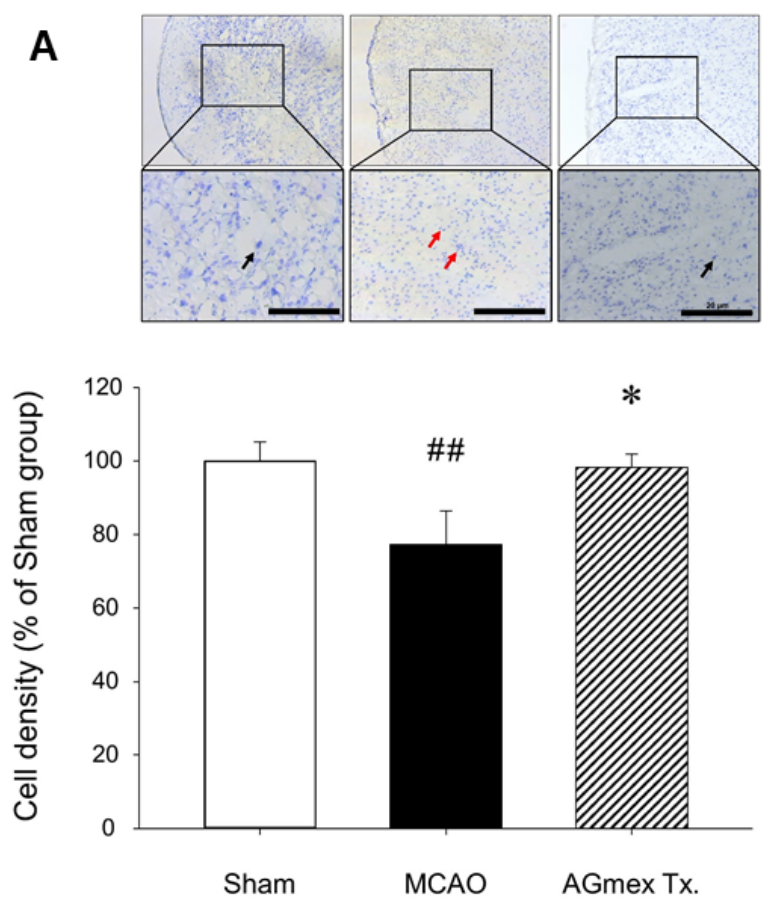

B
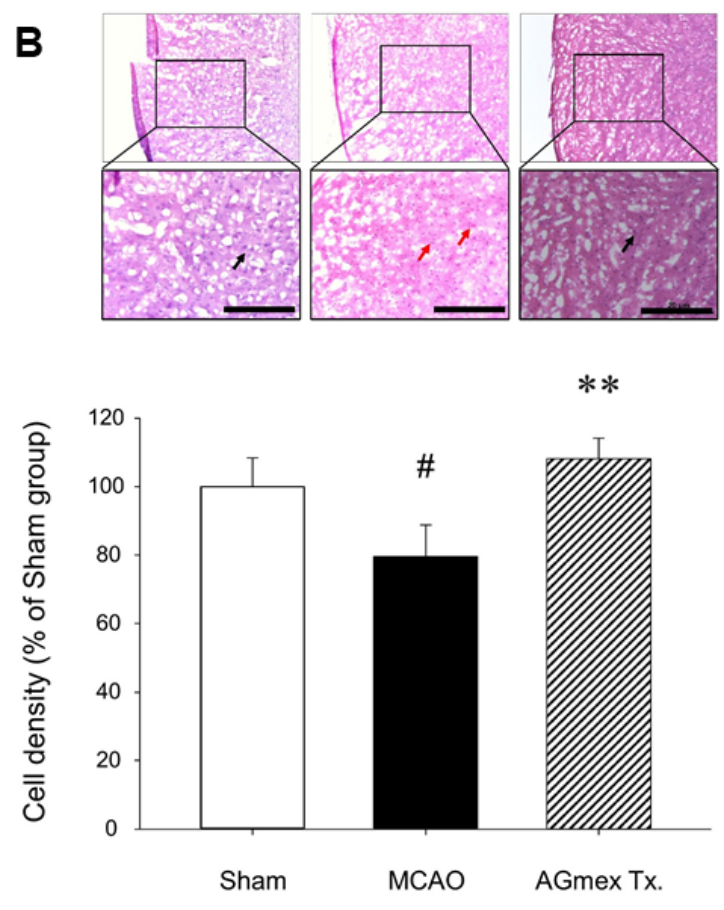

\section{Figure 5}

Neuroprotective effects of AGmex on MCAO-induced cell death. A, Representative photomicrographs of Nissl-stained neuronal cells in the subcortical area (upper images) and quantitative analysis showing changes in the number of neurons (lower graph). B, Representative photomicrographs of hematoxylin and eosin (H\&E)-stained neuronal cells (upper images) and quantitative analysis showing changes in the number of cells (lower graph). Black arrows indicate cells observed to be normally intact with wellarranged morphology, and red arrows indicate cells with apoptotic changes showing aberrant morphology. Scale bars: $20 \mu \mathrm{m}$. \# $p<0.05$, \#\# $\mathrm{p}<0.01 \mathrm{vs.} \mathrm{sham-operated} \mathrm{group;}{ }^{*} p<0.05,{ }^{\star *} p<0.01 \mathrm{vs}$. MCAO group. 
A

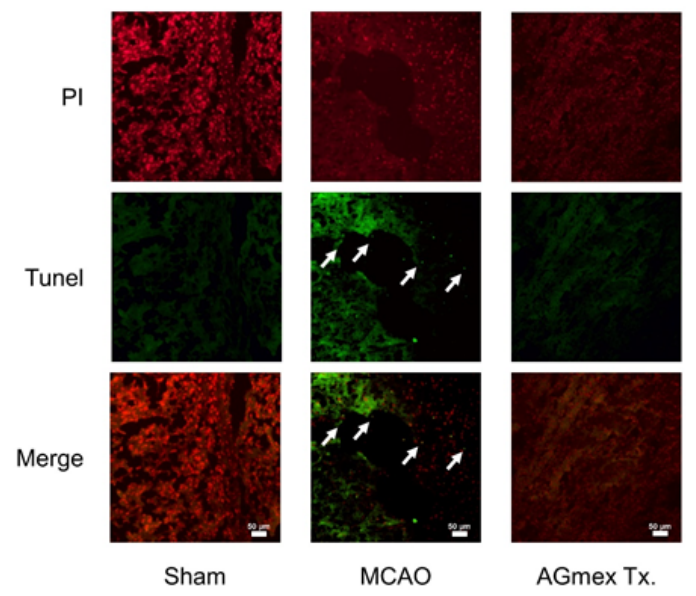

B

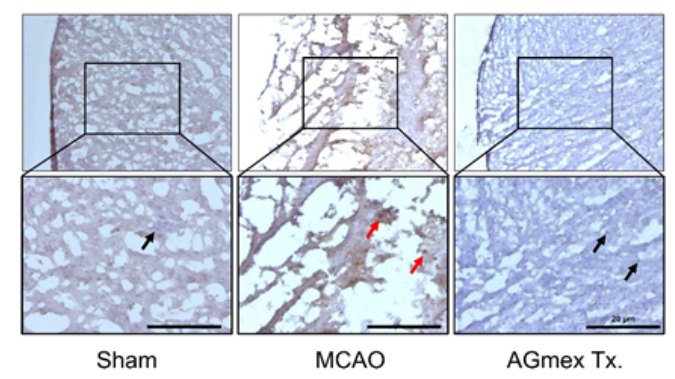

Figure 6

The effects of AGmex on MCAO-induced cell death. A, Representative images of TUNEL and PI doublestaining of MCAO-induced apoptosis in the cerebral cortex region; white arrows indicate cells with apoptotic change (scale bars, $50 \mu \mathrm{m}$ ). B, Representative images of IHC staining of MCAO-induced changes in mammalian target of rapamycin (mTOR) protein expression in the cerebral cortex region; red arrows indicate expression of mTOR and black arrows indicate mTOR negative stains (scale bars, 20 $\mu \mathrm{m})$. 


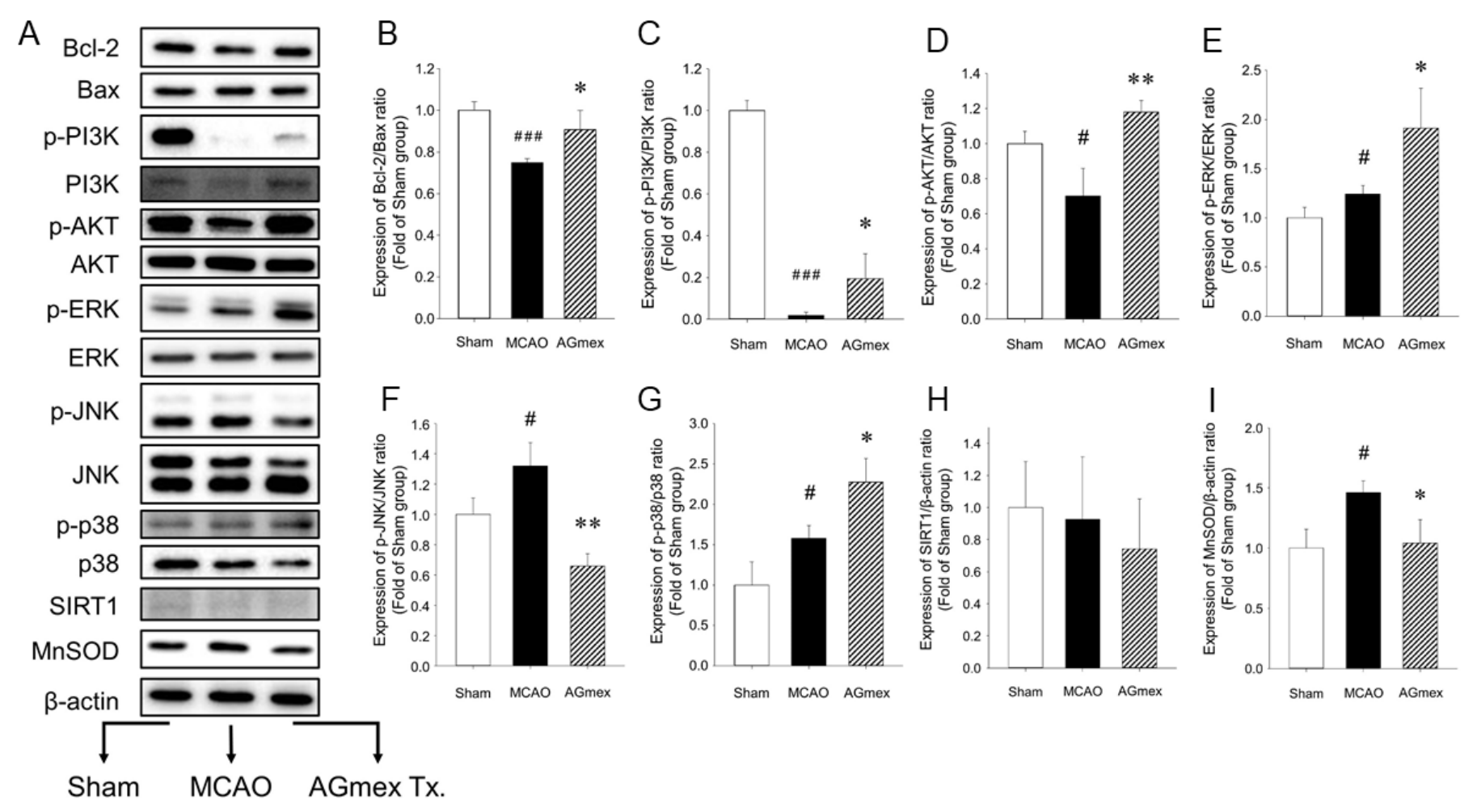

Figure 7

Effects of AGmex pre-treatment on proteins expressed in the MCAO-induced mouse brain. A, Representative image of western blot analysis of the expression of Bcl-2, Bax, p-PI3K, PI3K, p-AKT, AKT, pERK, ERK, p-JNK, JNK, p-p38, p38, SIRT1, MnSOD, and $\beta$-actin in the brain tissue. B-I, ratio of the expression levels of the various signaling proteins: B, Bcl-2/Bax; C, p-PI3K/PI3K; D, p-AKT/AKT; E, pERK/ERK; F, p-JNK/JNK; G, p-p38/p38; H, SIRT1/ $\beta$-actin; and I, MnSOD/ $\beta$-actin. All data are expressed as mean $\pm S D(n=3)$. \# $p<0.05$, \#\#\# $p<0.001$ vs. sham-operated group; * $p<0.05$, ** $p<0.01$ vs. MCAO group. 


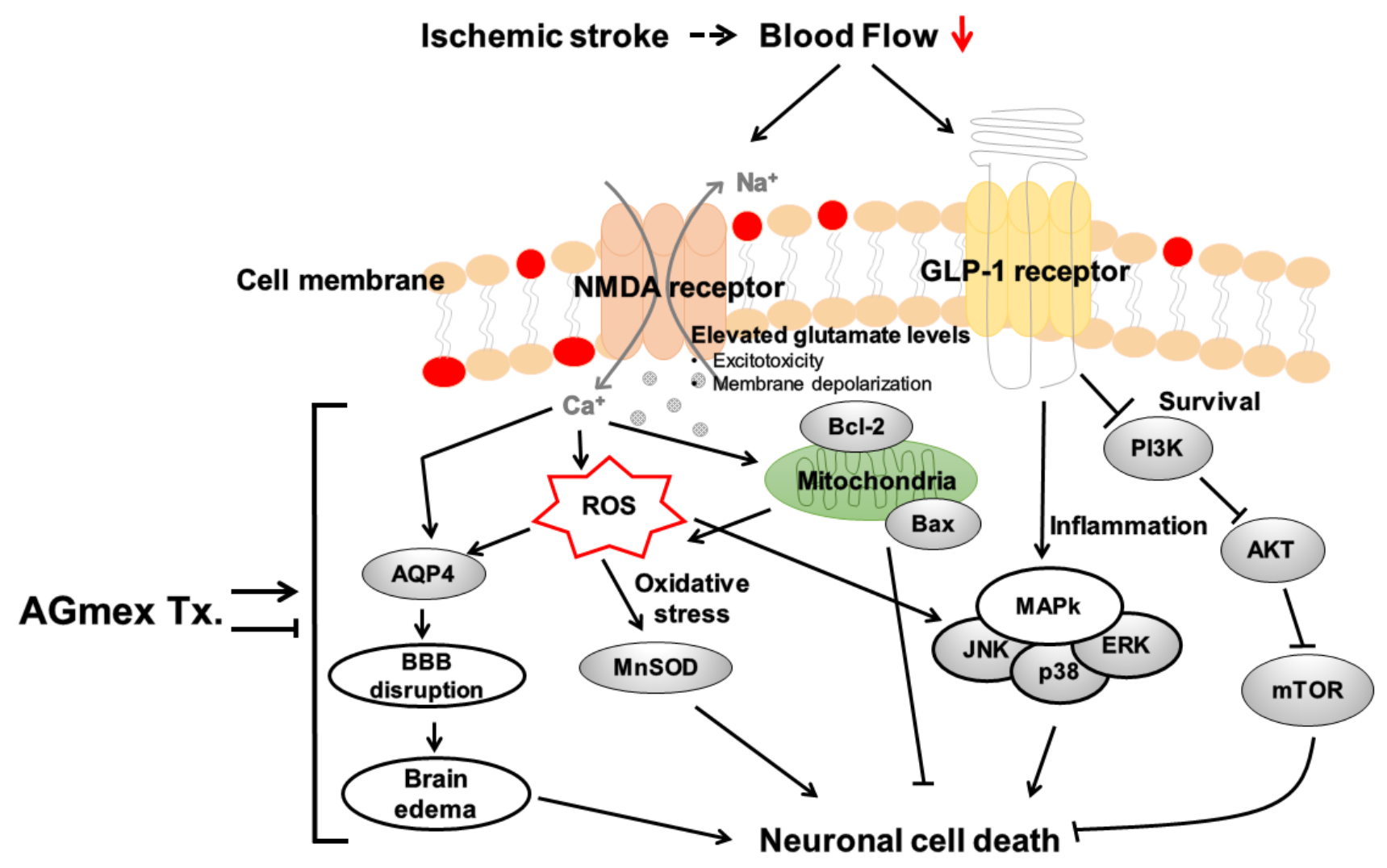

Figure 8

Schematic view of the cerebral damage pathways and neuroprotective mechanism of AGmex. The neuroprotective effect of AGmex was mainly related to anti-edema and anti-apoptotic pathways.

\section{Supplementary Files}

This is a list of supplementary files associated with this preprint. Click to download.

- AdditionalFile2.pdf

- GA.tif

- AdditionalFile1.docx 